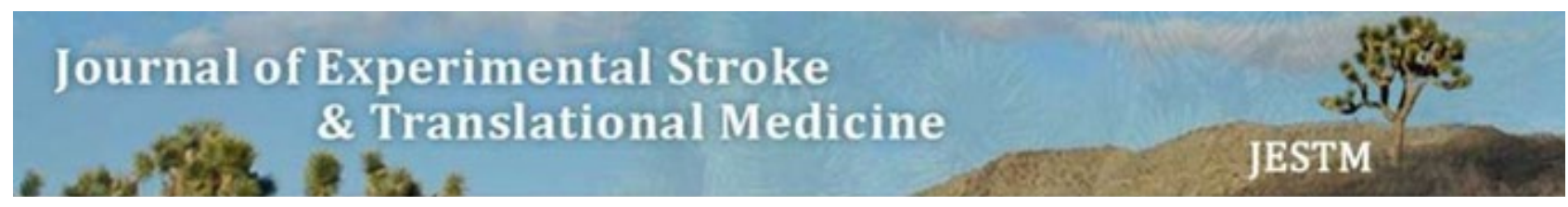

\title{
Carotid Vibro-Compression: A Reperfusive Treatment for Acute Ischemic Stroke? Feasibility Data and a Potential Risk / Benefit Discussion in View to Animal Testing
}

February 23, 2016 · Volume $9 \cdot$ Original Research

Andrew Hoffmann*, Harjit Gill

${ }^{1}$ Ahof Biophysical Systems Inc., Department of Research and Development, Burnaby B.C. Canada

${ }^{2}$ In-Vitro Laboratories, Burnaby, BC, Canada

Article Citation: Andrew Hoffmann, Harjit Gill. Carotid Vibro-Compression: A Reperfusive Treatment for Acute Ischemic Stroke? Feasibility Data and a Potential Risk / Benefit Discussion in View to Animal Testing. J Exp Stroke Transl Med. 2016 February. Online access at www. jestm.com

Correspondence should be sent to: Andrew Hoffmann, Ahof Biophysical Systems Inc., Department of Research and Development. 3858 Regent St., Burnaby B.C. Canada, Tel: 604779 3571, Fax: 604437 7961, E-mail: andrew.hoffmann11@gmail.com

\section{Abstract}

Slow uncertain reperfusion in Acute Ischemic Stroke (AIS) by intravenous thrombolysis has prompted search for alternative therapies. Pressure/flow disturbances from rapid compressions $(20-24 \mathrm{~Hz})$ of a clotted flow tube remote from thrombosis site have shown to assist reflow. Carotid Vibro-Compression (VC), a potentially risky maneuver, may therefore enhance AIS reperfusion but verification is needed that vibro-compressions would transmit flow disturbances to the cerebral arteries. From a pool of 15 volunteers (mean age 48) carotid compressions $(5$ to $8 \mathrm{~Hz},<=\sim 1 \mathrm{~cm})(n=11)$ and vibration $(30 \mathrm{~Hz} \sim 1 \mathrm{~mm})(n=10)$ were independently and together implemented with ipsilateral and contralateral Doppler flow monitoring of an intra-cranial artery. Doppler pulses from compressions were immediately demonstrated in all vessels $(26 / 26)$ under all conditions. Pulses from vibration were observable in a majority of vessels $(22$ / 25), but signals were often challenging to obtain. Mean intra-cranial flow velocities trended to increase during compressions $(46.1 \pm 6.4 \mathrm{~cm} / \mathrm{s}$ vs. $44.0 \pm 4.9 \mathrm{~cm} / \mathrm{s})$, but the difference was not statistically significant $(p=0.06)$. Carotid VC reliably transmits flow pulses to the cerebral vasculature, however vibration requires biofeedback to ensure device positioning. Carotid VC appears feasible for animal testing, but safety remains a major question.

Key Words: acute stroke; sonothrombolysis; carotid artery; vibration; compression, cerebral blood flow.

\section{Introduction}

Carotid vibro-compressive maneuvers are selectively utilized during Trans Cranial Doppler (TCD) examinations [Aaslid R.1986, Guan J et al.2013, Jawad Naqvi et al. 2013 ], however embolic plaque rupture leading to stroke in a neurologically symptomatic patient has since flagged caution with this technique [Khaffaf $\mathrm{N}$ et al. 1994]. Moreover Carotid Sinus Massage (CSM) and routine ultrasonic carotid imaging has been known in very rare incidences to induce stroke - with the latter occurring again in the neurologically symptomatic [Beal MF et al. 1981, Bastulli JA et al.1985, Friedman SG. 1990 ]. Carotid manipulations are therefore recommended only when absolutely necessary and following inspection of the carotid to be free of plaque [Aaslid R. 1986] - including high grade stenosis or ulcerating atheromata [Lupetin AR et al. 1995]. However, what if a patient is having a hot stroke already? What if the agitative hemodynamics of serially applied carotid deformations could expedite clearance of an acutely occluded cerebral vessel - perhaps within minutes or even seconds? This paper aims to introduce this subject, show feasibility towards animal testing, and offer a preliminary discussion on the potential risk / benefit regarding this controversial technique.

Intravenous (IV) recombinant tissue plasminogen activator (rt-PA) remains the only treatment approved for Acute Ischemic Stroke (AIS) in the 3 to $4 \frac{1}{2}$ hr window [N Engl J Med. 1995, Hacke W et al. 2008]. However half of rt-PA-treated patients remain severely disabled or die within 3 months with the major reason listed as a severe initial ischemic insult with slow and incomplete recanalization. Normalized cerebral flow has been quoted as only occurring $20-50 \%$ of the time within 2 hrs of thrombolysis [Pagola J et al. 2007], therefore alternative therapies to accelerate reperfusion are required.

Transcranial ultrasound has been studied to accelerate AIS thrombolysis however this technique has not yet translated in phase 3 clinical trials [Saqqur $\mathrm{M}$ et al. 2014]. Ultrasound has difficulties including a historic requirement for a skilled imaging approach to establish therapy (non-ideal for emergencies) [Alexandrov et al. 2004, Eggers J et al. 2003] and an increased risk of intra-cerebral bleeding when applied at higher power levels and /or lower frequencies [Eggers J et al. 2003, Daffertshofer M et al. 2005], which appear required to ensure therapeutic transcranial penetration [Pfaffenberger $S$ et al. 2005]. We have been studying an alternative premise that infrasonic to low sonic Vibro-Compressions (VC) may be useful to enhance reperfusion, due to VC's known clot disruptive [Hoffmann A et al. 2012, Yohannes FG et al. 2008, Wobser E et al. 1978, Folts J. 1991, Hoffmann A et al. 2012 ], and superior deep bodily penetration capabilities [Koiwa Y et al. 1997, Thomas J et al. 1995 ]. Moreover, VCs provide unique internal, transmission characteristics through tissue [Koiwa Y et al. 1986 , Hashiguchi R et al. 1988, Smith D et al. 1984 ] and along arteries [Farber JJ et al. 1963] which suggests a less imperative need for exact therapeutic targeting. Proposed VC action includes transmission of oscillations from an external body surface to a thrombosed artery to cause mechanical agitation of clot and blood proximate the clot, while also potentially inducing a localized vasodilation response to the culprit vasculature. Turbulence induced at the blood clot interface is predictive (as shown in-vitro [Hoffmann A et al. 2012]) and inferable (in accordance with hydrodynamic mixing studies [Hancil V et al. 1978, Neild A et al. 2010, Oberti S et al. 2009] to encourage systemic 
mixing of thrombolytics down an otherwise zero flow cerebral artery. Acoustic AIS therapy is generally problematic however as the cerebral arteries are mechanically shielded within the cranium. Recent work at Simon Fraser University (SFU, B.C. Canada) however has unveiled that rapid VC (20 to $24 \mathrm{~Hz}$ ) of a thrombosed flow tube even up to $60 \mathrm{~cm}$ remote from an occlusive thrombosis site greatly enhances reflow, with the reperfusive action attributed to a battery of pressure fluctuations propagating along the tube to agitate the clot [Marzencki $\mathrm{M}$ et al. 2012]. We have thereby resurrected a hypothesis that carotid VC may be useful in accelerating AIS reperfusion [Hoffmann A. 2010]. However, while carotid compressions are known to transmit flow pulses intra-cranially [Aaslid R.1986], before considering animal trials confirmation is required whether low sonic vibration would also propagate. Moreover information on the reliability of pulses propagating from the carotid (and particularly when delivered contra-laterally from the intra-cranial vessel) is scarce and in need of review. To this end we recruited 15 healthy volunteers for administration of ipsilateral and contralateral carotid deformations via fingertip $(5-8 \mathrm{~Hz}, 1 \mathrm{~cm}$ displacement) and with a vibrator $(30 \mathrm{~Hz}, \sim 1 \mathrm{~mm})$ while studying Doppler flow effects in at least one cerebral artery. Flow transmissions to the ipsilateral temporal artery, carotid siphon and internal carotid artery were also assessed.

\section{Materials and Methods}

The study was conceived in industry (Ahof Biophysical Systems Inc, Burnaby, B.C., Canada). The test subjects (the Authors and sampling of healthy volunteers) gave informed consent to the study which had been approved by Institutional Review [ABS Inc.: Ethics Review Board and Safety Committee] in accordance with the ethical standards on human experimentation per the Helsinki Declaration of 1975 , as revised in 2008.

\section{Choice of carotid "compression" and "vibration" parameters:}

A carotid compression frequency of 5 to $8 \mathrm{~Hz}(1 \mathrm{~cm}$ displacement) was chosen as it could be easily administered by finger-tip or manual oscillation of a vibratory instrument. To administer low sonic vibration we chose the Hitachi Wand (Hitachi Ltd., Tokyo, Japan), a device advertised for gentle massage including the neck and facial regions which in use with a power controller enabled sinusoidal emissions of $30 \mathrm{~Hz}(\sim 1 \mathrm{~mm}$ displacement). Thirty $\mathrm{Hz}$ was selected as a compromise between what SFU employed in their experiment [Marzencki M et al. 2012] and to frequency outputs utilized by thrombolysis catheters [Arko $F$ et al. 2007] and to those having demonstrated clot clearing effects in our lab [Hoffmann A et al. 2012]. Additionally low sonic frequency vibration is known to produce convection currents [Margulis $\mathrm{M}$ et al. 1982, Griesinger HR et al. 1989] and transverse wave propagation along the length of arteries [Farber JJ et al. 1963], hence is mechanistically predictive to provide an agitative, propagative effect. A 1-mm amplitude was deemed acceptable as given a typical carotid lumen diameter of 6 to $7 \mathrm{~mm}$ [Krejza $\mathrm{J}$ et al. 2006], the deformation would be predictive to compress the lumen by up to about 10 to $15 \%$ which was comparable to that utilized in the SFU study. For dual VC therapy (with $30 \mathrm{~Hz}$ vibration applied simultaneously with 5 to $8 \mathrm{~Hz}$ compressions), hand delivered oscillation of the Wand was simply utilized during vibrator activation.

\section{Choice of application site:}

We chose the distal Common Carotid Artery (dCCA) just under the chin within the carotid triangle were the internal and external carotid is expected to bifurcate (proximate the carotid bulb), to bring the generation site of hemodynamic pulses as close as possible to the intracranial vasculature.

\section{Choice of Doppler probes:}

Because of equipment availability we chose a cardiac phased array echo-transducer (S4 - Philips, and M5S GE) to perform intracranial arterial color and pulse wave Doppler interrogations. For Doppler evaluation of the extra-cranial arteries we used linear probes (L9-3 Philips, and 9L -GE).

\section{Protocol}

Fifteen healthy volunteers were recruited for assessment of flow transmissions induced by carotid VC as measured by pulse wave Doppler to at least one intra-cranial artery including the; proximal Middle Cerebral Artery (pMCA), distal Middle Cerebral Artery (dMCA) and/or Posterior Cerebral Artery (PCA). The ipsilateral Superficial Temporal Artery (STA) and time permitting the Internal Carotid Artery (ICA) and / or the Carotid Siphon (CS) were also interrogated. Due to ability to locate images, varying levels of consent, and time restraints the protocol was sometimes broken up with only selected parts performed with volunteers optionally returning at later dates for further testing. The test group included 8 males and 7 females (collective mean age 48 years), three subjects having a family history of coronary artery disease, and one with a personal history of mild hypertension. All test subjects were non-smokers and otherwise free of neurovascular disease or cardiovascular risk factors.

\section{Doppler assessment of intra-cranial cerebral arteries}

Firstly location of an intra-cranial artery was attempted via a phased array transducer placed upon the temple. Once a Doppler waveform was obtained the volunteers were instructed to palpate their ipsilateral carotid with their index and middle finger and then rapidly thrust their fingers in and out (up to about $1 \mathrm{~cm}$ ) about 5 to 8 times a second to serially compress / decompress the vessel'. Resulting flow disturbances were then recorded. A contralateral carotid was also compressed as a later addendum to the protocol.

Willing volunteers were then given our vibrator to practice carotid applications. The volunteers were shown to utilize a moderate, comfortable engagement force (i.e. about 10 to 20 Newtons - not expected to completely compress the vessel), with the Wand's contact node nestled within the carotid triangle, where-after an intra-cranial artery was Doppler recorded with carotid vibration. If no vibratory response was seen initially the volunteers were instructed to engage the Wand's contact node more forcefully and / or shift the device's position until vibratory pulses became evident. Then after a few seconds of recording the volunteers were instructed (sometimes with help from the researcher) to also rapidly oscillate the massager's handle (which in turn oscillated the contact node against the carotid) between 5 to 8 times a second during continued vibration. Data was collected as best as possible in view of occasional loss of imaging signal and within the confines of re-finding the cerebral artery (which was at times difficult to re-locate). A contralateral carotid was also vibrated as a later addendum to the protocol.

Following completion of the study five subjects were additionally assessed for left and right sided intra-cranial mean blood flow velocity

${ }^{1}$ We asked the volunteer's to perform their own neck compressions as this tended to ease their anxiety towards the procedure. 
immediately before versus initiation of ipsilateral carotid compressions (by comparison of heart rate indexed velocity time integrals during a cardiac cycle - end QRS complex to end QRS complex). For blood flow extrapolation we assumed that intra-cranial vessel diameter would remain constant. To assess whether there was a statistical difference between the groups we used the Wilcoxon Signed Rank Test (for comparison of two treatment populations) whereby a $P$ value of $<0.05$ was considered significant. Variability in the groups was reported by their standard deviation.

\section{Doppler assessment of extra-cranial vessels}

Secondly the procedure was essentially repeated but with a linear probe to obtain Doppler tracings of an ipsilateral STA. Time and volunteer permitting an ipsilateral ICA and CS were also interrogated. The STA was emphasized as being a superficial vessel the fidelity of the flow recordings would be optimized, and being of comparable distance from the carotid as the carotid is to the intracranial vessels may foresee ably be used as a biofeedback tool to ensure optimized carotid vibro-compression delivery.

An image of the vibro-compressive techniques are shown in (Figure 1).
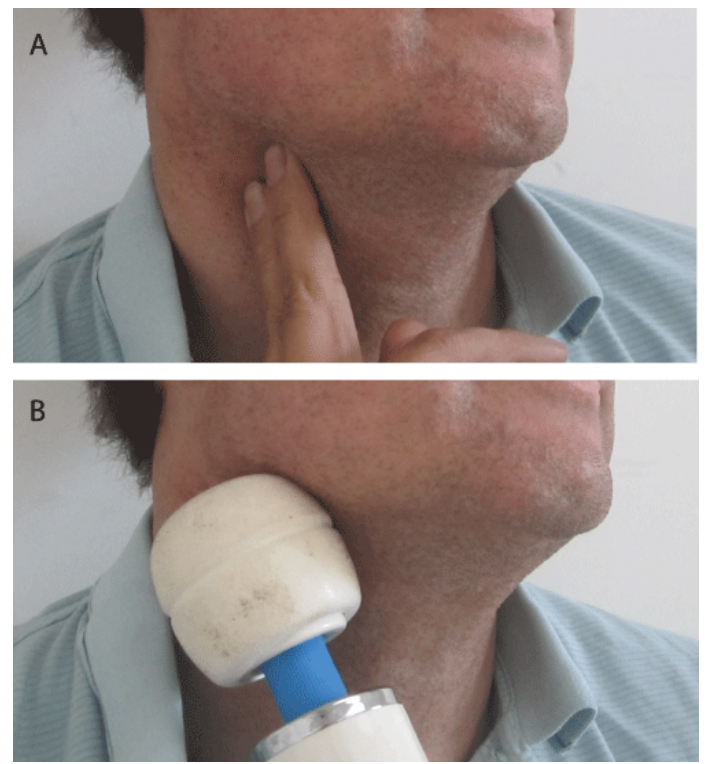

\section{Figure 1.}

Image (A) shows fingertip placement for carotid compressions. Image (B) shows application of the Hitachi Wand for applying vibration. Manual oscillation of the Wand's handle enabled "dual" VC therapy.

A movie showing carotid VC technique is available - see link at https://youtu.be/jlGqZjA8LXc

\section{Results}

\section{Doppler assessment of intra-cranial arteries}

Of the 15 test subjects 12 agreed to intra-cranial arterial ultrasonic interrogation, of which ultrasonic detection of at least one intra-cranial artery was possible in 11 total subjects.

Doppler flow waveforms concomitant with carotid compressions were assessed in 11/11 of these individuals (resulting in an assessment of 21 ipsilateral and 5 contra-lateral intracranial arteries).

Doppler flow waveforms concomitant with carotid vibration were assessed in 10/11 of these individuals (resulting in an assessment of 20 ipsilateral and 5 contralateral intracranial vessels).

\section{Carotid Compressions}

Marked flow fluctuations were immediately and reliably obtainable in all interrogated intra-cranial vessels (26 / 26) given compressions of an ipsilateral or contralateral carotid (i.e. $5-8 \mathrm{~Hz}$ ), regardless of how the compressions were applied (see Figure $2 \mathrm{~A}$ Vs. B, and $3 \mathrm{~A}$ ). 

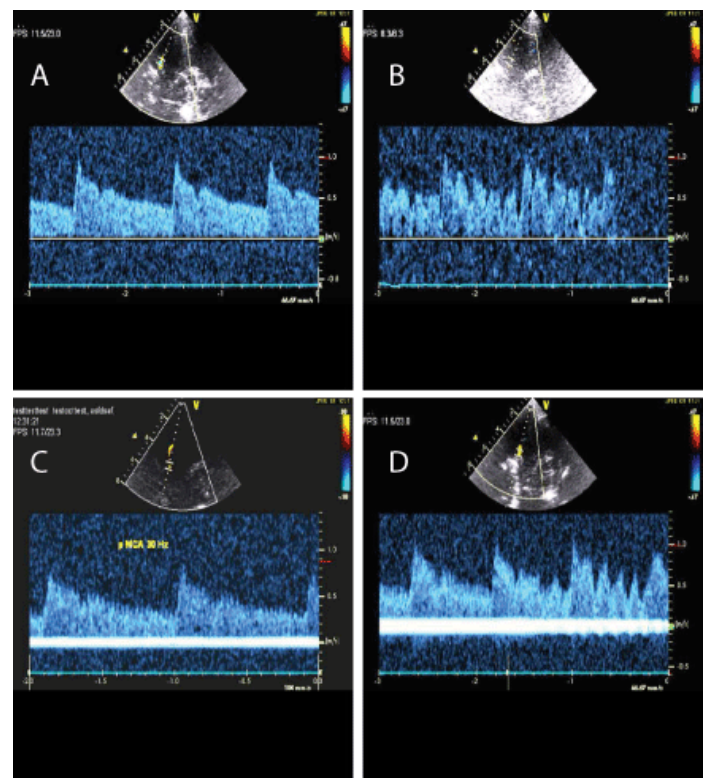

Figure 2.

Image (A) A pMCA Doppler trace. Image (B) Same pMCA Doppler trace with ipsilateral $5 \mathrm{~Hz}$ carotid compressions. Image (C) A pMCA Doppler trace with ipsilateral $30 \mathrm{~Hz}$ carotid vibration. Image (D)A pMCA Doppler trace with $30 \mathrm{~Hz}$ vibration followed by simultaneous $5 \mathrm{~Hz}$ Compressions - the "dual" response - applied from an ipsilateral carotid artery..

Contralateral applications subjectively yielded slightly dampened, fainter flow pulses relative to their ipsilateral counterparts.

\section{Carotid Vibration}

Vibratory flow fluctuations were obtainable in all but two ${ }^{2}$ (18 of 20) interrogated intra-cranial vessels given applications of $30 \mathrm{~Hz}$ upon an ipsilateral carotid artery (Figure $2 \mathrm{C}$ and D). However in the majority of cases (13/18) an observed vibratory response was not immediate and a degree of minor device adjustment (again in either its engagement force or positioning - often closer to the trachea) was required to obtain a vibratory signal. Following vibratory recording further demonstration of a dual flow response along with compressions was always obtainable (Figure 2 D - right half of image). Vibratory response in general at times however appeared somewhat transient, manifesting on and off and to varying degrees. We attributed this to vibrator placement - however varying quality of image or fidelity of the imaging probe also may have played a role. Carotid vibration was also shown to transmit contra laterally in 4 of $5^{3}$ test subjects (as exemplified in Figure $3 \mathrm{~B})$, however transmissions appeared in most cases substantially fainter, and took more time to obtain.
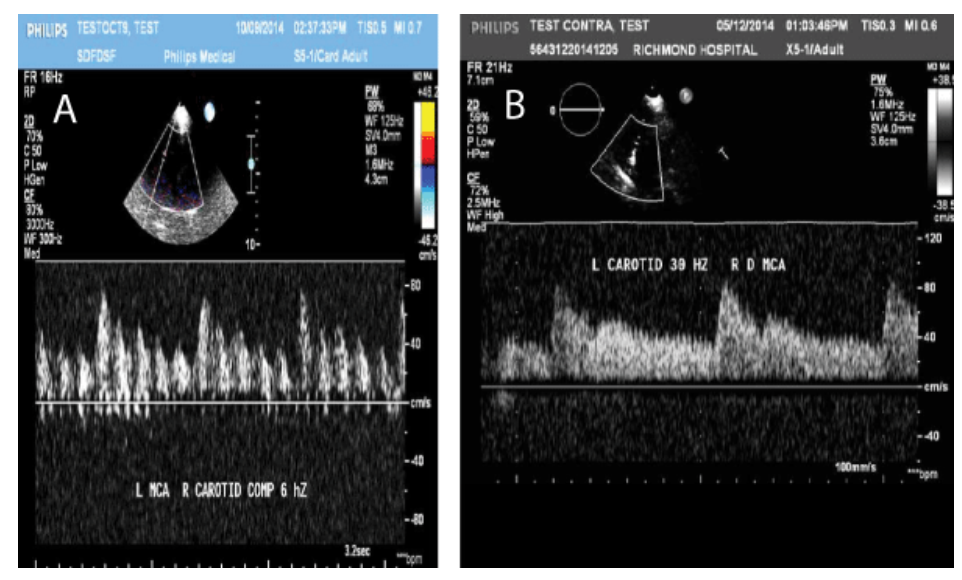

\section{Figure 3.}

Image (A) A left pMCA Doppler trace with $6 \mathrm{~Hz}$ contralateral right carotid artery compressions. Image (B) A right dMCA trace with $30 \mathrm{~Hz}$ contralateral left carotid vibration.

${ }^{2}$ In the first subject only a brief vibration attempt was made along with a PCA Doppler trace where-after the image was lost and could not be regained. In the second subject a clear MCA vibratory response could not be observed despite two minutes of vibrator repositioning.

${ }^{3}$ In one test subject a vibratory response could not be observed in a contralateral pMCA segment despite two minutes of vibrator repositioning. 
Further imaging examples showing the Doppler flow response of ipsilateral carotid VC to the dMCA and PCA are shown in (Figure 4).
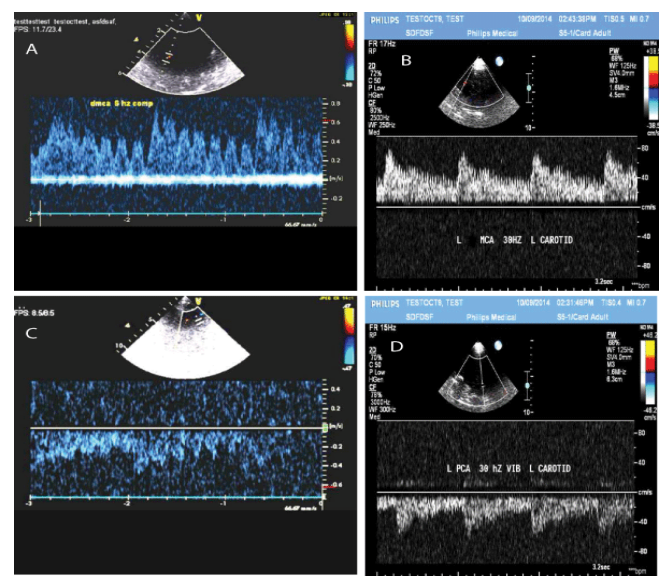

Figure 4.

Image (A) A dMCA Doppler trace with $6 \mathrm{~Hz}$ ipsilateral carotid compressions. Image (B) A dMCA Doppler trace with $30 \mathrm{~Hz}$ ipsilateral carotid vibration. Image (C) A PCA Doppler trace with $5 \mathrm{~Hz}$ ipsilateral carotid compressions. Image (D) A PCA Doppler trace with $30 \mathrm{~Hz}$ ipsilateral carotid vibration.

\section{Intra Cranial Mean Blood Flow Velocity}

In all five assessments the onset of carotid compressions were marked by an immediate change in the intra-cranial arterial doppler waveform with the appearance of a battery of upstrokes in the flow pattern apparent throughout the cardiac cycle. Flow velocities during both systolic and diastolic compression cycles at times exceeded non-compressed systolic peak velocities, and generally demonstrated marked, increased pulse pressures including at times transient loss of diastolic flow. Mean arterial flow velocities tended to increase during initiation of compressions $(46.1 \pm 6.4 \mathrm{~cm} / \mathrm{sec})$ vs. their immediately prior non-compressed states $(44.0 \pm 4.9 \mathrm{~cm} / \mathrm{sec})$, but this trend did not reach statistical significance $(p=0.06)$. Figure 5 for a sample of pre versus initiation of ipsilateral carotid compressions in a Doppler MCA waveform. Table below for a summary of intra-cranial mean blood flow velocity in five subjects.

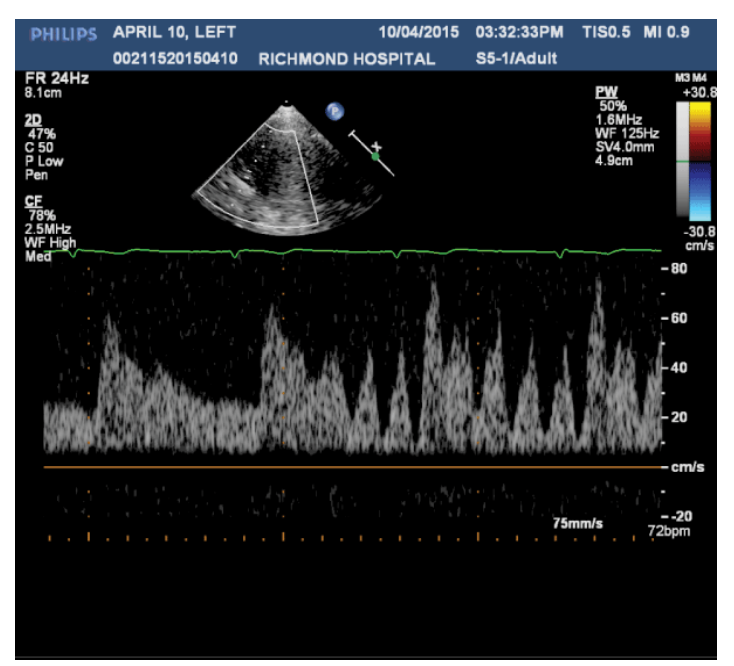

Figure 5.

An MCA Doppler trace showing initiation of carotid compressions. Mean flow velocity was $33.9 \mathrm{~cm} / \mathrm{sec}$ just prior (first cardiac cycle) and $37.0 \mathrm{~cm} / \mathrm{sec}$ during compressions (third cardiac cycle) in this sample.

\begin{tabular}{|c|c|c|c|}
\hline Subject & Vessel & No Compressions (cm/sec) & Compressions (cm/sec) \\
\hline 1 & L MCA & 42.8 & 45 \\
\hline & R MCA & 44.5 & 50.5 \\
\hline & L MCA & 46.4 & 47.2 \\
\hline 3 & R MCA & 44.9 & 50.7 \\
\hline 4 & L MCA & 48 & 52.9 \\
\hline
\end{tabular}




\begin{tabular}{|c|c|c|c|}
\hline & R MCA & 49.6 & 47.3 \\
\hline 5 & L MCA & 33.9 & 37 \\
\hline & R MCA & 37.3 & 35.2 \\
\hline Average +/- SD & & $44.0 \pm 4.9$ & $46.1 \pm 6.4$ \\
\hline
\end{tabular}

\section{Table 1.}

Mean Intra-cranial Doppler Flow Velocities with and without Carotid Compressions.

\section{Doppler assessment of the extra-cranial arteries (STA, ICA and CS)}

Of the 15 test subjects 14 agreed to ipsilateral extra-cranial vessel Doppler interrogation, of which ultrasonic detection of at least one extra-cranial artery was possible in all 14 subjects.

Doppler flow waveforms concomitant with carotid compressions were assessed in 14 / 14 of these individuals for a total evaluation of flow disturbances to the STA (14), CI (5), and CS (4) - for a total of 23 ipsilateral extra-cranial arteries interrogated.

Doppler flow waveforms concomitant with carotid vibration were assessed in 12 / 14 of these individuals for a total evaluation of flow disturbances to the STA (12), $\mathrm{Cl}(5)$ and CS (4) - for a total of 21 ipsilateral extra-cranial arteries interrogated..

\section{Carotid Compressions}

Marked flow fluctuations were reliably and immediately observed in all extra-cranial vessels (23 / 23) given serial compression of an ipsilateral carotid artery $(\sim 5-8 \mathrm{~Hz},<=1 \mathrm{~cm})$, regardless of the compressive technique (see Figure 6).
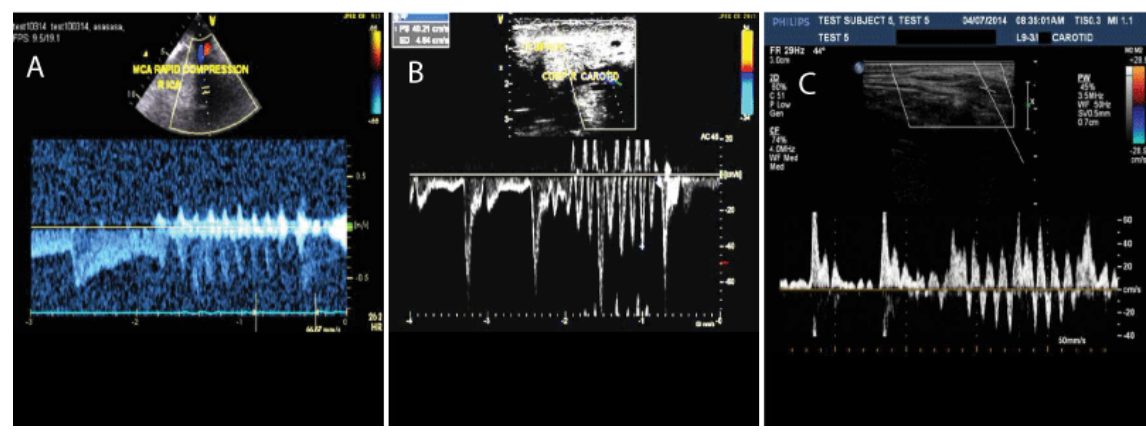

\section{Figure 6.}

Image (A) ICA Doppler trace followed by $\sim 8 \mathrm{~Hz}$ ipsilateral carotid compressions. Image (B) CS Doppler trace followed by $\sim 7$ $\mathrm{Hz}$ ipsilateral carotid compressions. Image (C) Right STA Doppler trace followed by $\sim 5 \mathrm{~Hz}$ ipsilateral carotid compressions.

\section{Carotid Vibrations}

Vibratory flow fluctuations were observed in all interrogated vessels $(21 / 21)$ given application of $30 \mathrm{~Hz}$ upon an ipsilateral carotid (Figure 7). However, in 10 applications at least a few seconds of minor adjustment of the vibrator's contact node in either positioning, or via a slightly increased (and rarely decreased) engagement force, was first required to obtain a vibratory signal. After successful vibratory recordings all cases additionally demonstrated a dual Doppler flow response of vibration along with carotid compressions (Figure 7 D).

The above vibro-compressive techniques were described in all cases as reasonably comfortable and absolutely without pain. However a mild "gag" or "choking" sensation was occasionally mentioned in four volunteers when the Wand's contact node was engaged too forcefully or close to the trachea. Furthermore the Author $(\mathrm{AH})$ noticed at times a mild, asymptomatic transient reduction of heart rate during employment of the Wand upon his carotid artery. 


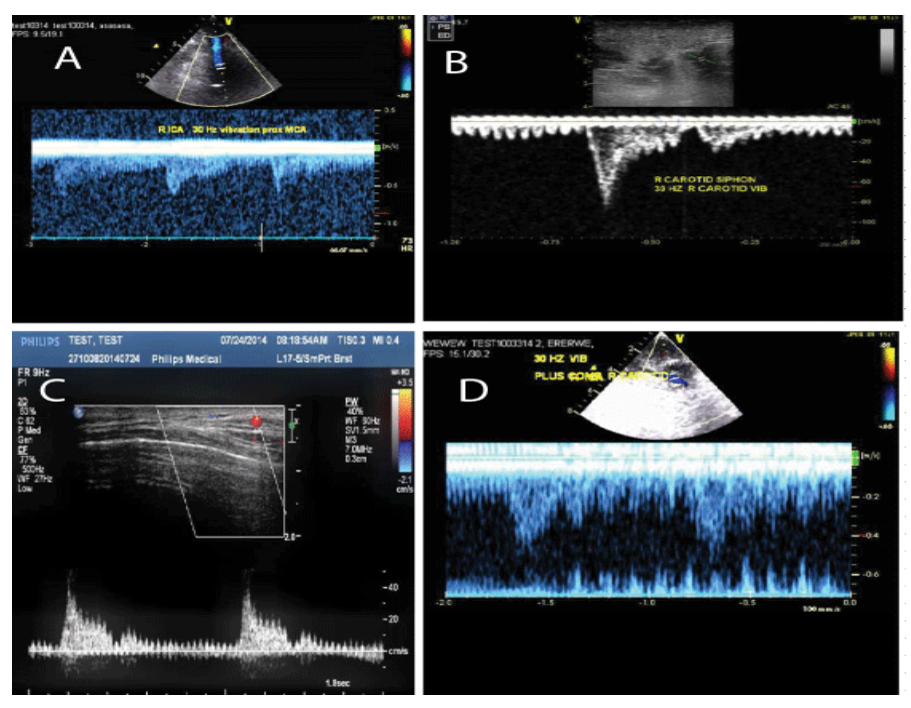

Figure 7.

Image (A) ICA Doppler trace with $30 \mathrm{~Hz}$ ipsilateral carotid vibration. Image (B) CS Doppler trace with $30 \mathrm{~Hz}$ ipsilateral carotid vibration. Image (C) STA Doppler trace with $30 \mathrm{~Hz}$ ipsilateral carotid vibration. Image (D) STA Doppler trace with $30 \mathrm{~Hz}$ vibration along with $5 \mathrm{~Hz}$ ipsilateral carotid compressions.

\section{Discussion}

Just as a plunger is often a first measure in unblocking a toilet we hypothesize that the repetitive compression and suction aspects of hemodynamic fluctuations transmitted from a vibro-compressed carotid may promote clearance of an acutely obstructed intra-cranial cerebral artery. This study confirms that non-invasive serial carotid compressions (i.e. 5 to $8 \mathrm{~Hz},<=1 \mathrm{~cm}$ ) with a high reliability, and carotid vibrations (i.e. $30 \mathrm{~Hz}, 1 \mathrm{~mm}$ ) with at least a transient reliability, transmit flow pulses to the intra-cranial arteries in live test subjects. This demonstrates feasibility for the technique in pursuit of AIS studies, however the prospective safety of the technique (which is otherwise deemed a risky maneuver in neurologically symptomatic individuals) must first be addressed.

\section{Safety Considerations}

For ethical /safety reasons our study was limited to the healthy middle aged (max age 64), hence we could not assess the prospective safety of carotid VC in a population comparable to AIS patients.

A risk assessment in applying carotid VC for AIS is at this point entirely speculative. Nevertheless a preliminary safety discussion is possible based on experience in carotid vibro-compressive maneuvers during neurovascular therapy, TCD testing, Carotid Sinus Massage (CSM), and head / neck trauma. We also consider prior work with External Counter Pulsation (ECP) for AIS - which similar to carotid VC has its therapeutic action by transmission of pressure/flow pulses to the cerebral vasculature. We have divided our safety discussion based on; embolic risk, hemodynamic risk, risk of intracerebral bleeding, and risk of carotid dissection.

\section{Risk of Embolus.}

We applied VC proximate the carotid sinus to bring the site of pulse generation as close as possible to the intra-cranial vasculature. However isolated incidents of cerebral embolism from ulcerated plaques has been noted following carotid compression [Khaffaf $\mathrm{N}$ et al. 1994], especially over the carotid sinus [Beal MF et al. 1981, Bastulli JA et al. 1985].

Carotid sinus compression in the elderly with a high incidence of atherosclerosis was assessed in 502 patients whereby the complication rate with lasting sequale was reported at less than $0.5 \%$ [Lacerda $G$ et al. 2009]. Also the risks of CCA compressions during indicated TCD (primarily in elderly, neurologically symptomatic individuals) was assessed in 3383 patients whereby less than a $0.4 \%$ risk of "brief, transient cerebral symptoms" (only during the CCA compressions), and just one probable Transient Ischemic Attack (TIA) [Jatuzis D et al. 2000] was documented. Furthermore repeated, daily carotid compressions have found prescription for treatment of Cavernous Sinus Dural Arteriovenous Fistulae (CS-DAF) in a variety of patients, including the elderly [Kai Y et al. 2007, Higashida RT et al. 1986].

While these reports suggest carotid compressions may be relatively safe, we stress that our proposed technique comprises a more vigorous and prolonged procedure, and in expressly neurologically symptomatic patients. For these reasons a requirement in treatment of stroke (such if carotid VC were investigated clinically), would most certainly involve prior imaging of a carotid planned for manipulation to rule out potential embolic risk, including significant carotid stenosis, ruptured or mobile plaque, dissection, and particularly clot ${ }^{4}$.

In perspective however it is worth noting that between 14 to $30 \%$ of AIS presentations are reportedly cardio-embolic [Arboixa A et al. 2010 ], and cerebral arterial in-situ thrombosis (rather than carotid embolus) is reported to account for a majority of AIS presentations in

${ }^{4}$ Animal studies [Folts J, 1991] have shown that external tapping of an acutely clotted carotid artery causes near immediate clearance of clot. Carotid thrombosis following AIS is an infrequent but potentially ominous finding, having a prevalence of $\sim 1-6 \%$ [Vellimana AK et al. 2013]. 
select subgroups (e.g. Asians, Hispanics and Africans) [Arenillas JF. 2011, Bum JK et al. 2014]. This predicts a large cross section of the population who may have a disease free carotid potentially available for VC therapy.

We should also stress that while it would seem advantageous to have pulse generation as close as possible to the obstructed vasculature, we have also shown that flow pulses transmit contra-laterally whereby a contralateral carotid (to the obstructed cerebral vessel) would be more likely free of unstable disease and thereby a possible treatment option. It is also worth mention that it is not at all clear whether VC of even an ipsilateral culprit "acutely neurologically active" carotid may still yield a fruitful risk / benefit outcome in an otherwise severely disabling stroke.

\section{Risk of Hemodynamic Decompensation}

A further safety concern in Carotid VC is that pressure applied to the carotid sinus could induce a vagal response including dangerous bradycardia (including heart block, sinus pauses, sinus arrest etc.), especially in the elderly who are the most common stroke victims. To this end cardiac monitoring and an IV would be required (for administration of fluids, vagalytics etc.), and optionally external pacing pads applied (with a defibrillator on stand by ${ }^{5}$ ), should this technique ever be tested clinically in acute stroke victims. Of note if offensive bradycardia were to occur the vibro-compression point could be attempted more gently, or slightly lower nearer the base of the neck to the proximal CCA in avoidance of the sinus.

\section{Risk of Intra-Cerebral Hemorrhage (ICH)}

Perhaps a premier concern for carotid VC therapy may be whether the battery of transmitting pressure pulses could increase the risk of Intra-Cranial Hemorrhage $(\mathrm{ICH})$. Cerebral bleeding has been a significant concern in administration of transcranial ultrasound assisted AIS thrombolysis in the TRUMBI trial (where the trial had to be discontinued because of increased ICH) [Daffertshofer M et al. 2005], and shows documented risk even without mechanically assisted measures in IV thrombolysis for ST Elevation Myocardial Infarction [Gurwitz A et al. 1998].

To address this issue we discuss ECP as a treatment strategy for AIS - a procedure somewhat similar to carotid VC (in the prorogation of hemodynamic pulses) but applied at a lower compression frequency and more peripherally to the arms or legs of patients. ECP for AIS has been reviewed by Han et al [Han J et al. 2008], who cite 22 Chinese papers since 1980 all of whom report a generally favorable clinical outcome with no reported serious adverse advents. Han [Leung T et al. 2008] since performed a randomized crossover assessmentblinded proof of concept trial in use of ECP for recovery of 50 AIS patients (weeks 1 to 7 , and 7 to 14 following stroke), whereby favorable clinical benefits and again no increased risks for $\mathrm{ICH}$ or any serious adverse events were documented. Galuma et al [Alexandrov A W et al. 2004] has most recently provided ECP study results on a randomized patient blinded trial in MCA strokes arriving ineligible for reperfusion within 48 hours of symptom onset (13 treatment, 10 sham) whereby a single ECP treatment session was performed. The Galuma study showed a lack of clinical improvement in the ECP group, but again from a safety perspective no increased risks for ICH, subsequent ischemic strokes, or death was reported. Other's have recently reported that prolonged, multiple daily ECP sessions over the first weeks of stroke tend to yield enhanced recovery benefits, and again with no reported increases of risks [Lin W et al. 2013, Li X et al. 2014].

The Author's caution however that while it appears that ECP has generally been deemed "safe" following AIS, none of the ECP trials have been run early in the hyper-acute stage of AIS (i.e. in association with IV thrombolysis), and the hemodynamic fluctuations transmitted by ECP are generally of lower amplitude and frequency in comparison with what would be expected in carotid VC. To this end, risk of ICH secondary to carotid VC, especially if run in conjunction with IV thrombolysis, remains a major question.

\section{Risk of Carotid Dissection}

Internal Carotid and Vertebral Arterial Dissection (ICAD and VAD respectively) with subsequent stroke are known risk factors for patients experiencing traumatic injury to the head or neck [Haneline MT et al. 2003, Cassidy JD et al. 2008, Wynd S et al. 2013]. Moreover, there is at least a temporal correlation between ICAD and chiropractic neck manipulations, although a clear causative link has and continues to be strongly argued against or at least questioned [Biffl WL et al. 1998, Norris JW et al. 2000, DeSouza RM et al. 2011]. Identified risk factors for Cervical Arterial Dissection (CAD) have been reported as abrupt hyper neck; flexion, extension, and particularly rotation - or more generally "sudden head movement" - all of which imply a history of sudden and forceful stretching, twisting or tortion of the dissected vessel [Haneline MT et al. 2003, Cassidy JD et al. 2008, Wynd S et al. 2013].

While VC therapy near the carotid triangle would certainly stimulate the CCA and ICA, the arteries would not be expected to be abruptly torqued or stretched comparably to the typical head/neck trauma or chiropractic scenario. Moreover as mentioned earlier the reported safety of CSM in the elderly with known carotid disease [Lacerda G et al. 2009], and CCA compressions as a diagnostic tool for indicated TCD [Jatuzis D et al. 2000], suggests some level of re-assurance that controlled inward compressions of even a plaque ridden carotid may be relatively safe. Never-the-less the Author's have mandated that an imaging inspection of any artery of the neck should be performed prior to commencement of VC therapy to rule out plaque, stenosis and evolving dissection (with or without clot). Certainly if future animal work were to be considered histological analysis of the carotid vessels should be performed following oscillation therapy to inspect for new dissection, however there is no strong evidence to suggest this would be an expected concern in healthy vessels.

\section{Proposed Procedure Length and Therapeutic Window.}

It is difficult to recommend the duration of a VC carotid therapy session in treatment of AIS. SFU showed a $75 \%, 90 \%$ and $100 \%$ recanalization rate in their flow tube within 5, 10 and 20 minutes of $\mathrm{VC}$ respectively [Marzencki M et al. 2012], hence perhaps in-vivo sessions of up to 20 minutes, or until obvious evidence of reperfusion would comprise reasonable end points. With regards to therapeutic window we propose carotid VC to commence as early as possible preferably at time of IV thrombolysis, as this would likely carry the greatest benefit particularly as vibration is known to potentiate thrombolysis in-vitro [Hoffmann A et al. 2012] and in catheter use for treatment of deep vein thrombosis [Arko $\mathrm{F}$ et al. 2007] ${ }^{6}$. However brain tissue is arguably salvageable within the first 24 hrs following AIS [Baron Jb et al. 2000], hence the benefit of any mechanical intervention which could potentially clear embolic debris within 24 hrs of symptom onset would be of interest for study.

${ }^{5}$ Extremely rare incidences of ventricular fibrillation during carotid sinus massage have been documented [Deepak S M et al. 2005]. ${ }^{6}$ Also see u-tube link on action of the Trellis ${ }^{\mathrm{TM}}$ Catheter system at https://www.youtube.com/watch?v=qgkOgWV2Frk 


\section{Feasibility of Carotid VC for Clinical Study}

In study of VC therapy there is a presumed need for determining a neurologically stable carotid in conjunction with a diagnosis of ischemic (vs. haemorrhagic) stroke. Recent trends for employing mobile CT units in ambulances for acute stroke calls [Silke W et al. 2012] could foresee ably provide these services. Disease free carotids could also of course be determined by routine ultrasound inspection in ambulance or in hospital.

\section{Limitations of our study}

One particular drawback to our study was that flow pulse transmission was only shown in a younger test group most of whom would have relatively compliant arterial walls. We did not see that as a major problem however, as pulse wave propagation in older, stiffer, atherosclerosed vessels is only known to differ by increased velocity, higher pressures and an increased pulse pressure (by the "Windkissel effect")[ Belz GG. 1995] - phenomena not expected to impact our results. Also low sonic vibration transmission efficiency has been shown as superior along non-compliant vs. compliant tissue [Smith D et al. 1984], and lower frequency hemodynamic transmission from carotid vibro-compressive maneuvers including in the elderly have of course been well documented [Aaslid R.1986]. Nevertheless, further study of flow pulse propagation in an older test population would be valuable to confirm the effect and for ongoing safety assessment.

Another drawback was that we only studied a distal carotid VC point substantially over the carotid sinus, whereby in hindsight (in light of safety concerns) the proximal CCA, although slightly further from the target vasculature, may have been a better choice. We have found $\mathrm{VC}$ of the proximal CCA can also transmit flow pulses intra-cranially ${ }^{7}$, although statistical validation of this technique is required.

We also did not study flow transmissions from the superficial V3 segment of the vertebral artery to the basilar artery, which (as the immediate proximal vessel to the basilar artery) would comprise a preferred treatment strategy for acute basilar arterial occlusions. The V3 segment is easy to palpate (even in obese patients), and should be included in future studies.

Finally while we showed a positive trend for increased mean intra-cranial flow velocity upon onset of carotid compressions, our sample size was small and the comparison of velocity time integral measurements carry inherent uncertainty based on variance in position of the pulse wave sample gate and quality of signal. Moreover the net increases in flow velocity observed in the compression group were modest (and were not uniform in all subjects), and straightforward extrapolation of velocity increases to actual blood flow increases (particularly in AIS cases), cannot be assured by our methods. In view of these considerations it is probably most prudent to state that carotid VC does not at least appear to substantially diminish cerebral arterial blood flow in a majority of healthy subjects.

We also did not study mean intra-cranial flow velocities with vibration as it was clear from our work that (and unlike with carotid compressions) any changes to the arterial Doppler waveforms were at most very slight and likely un-measurable given the limited fidelity and fore-mentioned confounding factors with respect to our measuring technique. Moreover any changes in velocity with vibration could be arguably attributed to a possible acute vasodilatory response within the stimulated intra-cranial artery (not unexpected with high frequency oscillation stimulus - by for example a hypothesized stimulation of nitric oxide release within the vessel [Maloney-Hinds $\mathrm{C}$ et al. 2009, Pei $Z$ et al. 2004, Aelen $P$ et al. 2010]) which, with a possible change in intra-cranial arterial lumen diameter, would confuse the correlative meaning between Doppler velocity and cerebral blood flow.

\section{Future Work}

\section{Animal Thrombolysis Study}

As a next step we recommend pre-clinical testing of carotid VC via a rabbit AIS model with speed and quality reperfusion gauged by serial cerebral angiography. Following selective MCA thrombotic occlusion an ipsilateral stenosis free carotid would receive VC therapy versus control. Preferably one research arm should include systemic IV thrombolysis to evaluate possible potentiation of the fibrinolytic effect. Confirmation of effective VC delivery (in generating hemodynamic pulses) should be performed by invasive pressure monitoring in conjunction with non-invasive Doppler and / or tonometry evaluation of a relevant downstream cerebral vessel. VC administration could be performed via the Hitachi Wand (or other comparable vibration instrument) with the engagement force against the carotid adjusted to that required to provide optimized propagating hemodynamic fluctuations. Alternatively a vibrator could also be fastened to the backside of an operator's hand (instruments such as this are known in the field of chiropractic and hairdressing) to thereby vibrate the operator's fingers, which in turn could reliably palpate and compress the carotid artery. We recommend treatment durations of 20 minutes or until TIMI 3 flow is achieved, with heart rate and systemic blood pressure monitored to assess for hemodynamic including vagal effects. Future work may consider manipulations of the proximal CCA in avoidance of the coronary sinus. Post procedure histological examination (e.g. 24 hours after stroke) should be performed to assess infarct size, secondary embolus, ICH and presence of carotid arterial dissection between the study groups. An exemplary rabbit model satisfactory to the above requirements has been established by Jahan et al [Jahan R et al. 2008].

\section{Study of optimized VC parameters}

Parameters for VC therapy include: administration site, mode of delivery, impact frequency, percussive wave-shape, displacement amplitude, vibratory pattern, engagement force of a vibratory instrument, resultant hemodynamic effect of pulses within the cerebral vasculature, and time spent undergoing the procedure.

It has been proposed by SFU that randomizing the timing and / or wave-shape of VC may be helpful in adding a more disruptive turbulence into the vasculature system [Menon C et al. 2008]. Indeed the assessment of timing VC to the beat of music (such as common to vibroacoustic therapy systems) may also be of interest. Of note Antic et al [Antic S et al. 2008] have shown an increase in MCA flow in sub-acute AIS victims while listening to MOSART sonatas.

Moreover vibration could potentially induce a vasodilatory response [Lindblad LE et al. 1986, Ljung B et al. 1975, Hudlicka O et al. 1978], and a possible endogenous increase in blood fibrinolytic activity [Takashima $\mathrm{H}$ et al. 1987]. Both of these factors could be studied in animal stroke models. Finally it would be interesting to assess the value of carotid VC administered selectively during the diastole of the cardiac cycle, which may provide (like ECP) an enhancement in cerebral blood flow in AIS therapy.

${ }^{7}$ The Author's have observed successful VC flow/pulse propagation from the proximal CCA to the intra-cranial cerebral vasculature informally (in study between themselves). 
We should note that there has been no established methods to empirically quantify the delivered hemodynamic pulses (whether by compression or vibration) - which may be needed to standardize a safe and effective "dosage" for future therapy. In our study we simply evaluated whether we were able to witness a compressive or vibratory undulation in the interrogated downstream arterial waveforms. This issue is not unique in the pursuit of a non-invasive mechanical methods for enhancing cerebrovascular reperfusion or thrombolysis, as the "dosages" delivered by transcranial ultrasound for example are defined entirely by their output frequency and power - and not by their resultant invasive effects (which would vary from patient to patient).

As Doppler monitoring of downstream cranial vessels may be a future way of ensuring delivery of carotid VC therapy, one option to define dosage may be to develop a grading scale whereby for carotid compressions a Doppler effect of; a) decompression waves resulting in flow reversal - would be considered "high intensity" (see Figure 6, B and C ), b) compression waves greater or equal to intrinsic systolic waves - "moderate intensity" (see Figure 5), and c) compression waves of lesser size than systolic wave - "mild intensity" (see Figure 3 A); could be implemented. A practical grading scheme for vibration delivery however may be more difficult as the effects, when noted, seem quite similar between samples. For now a simple "yes" or "no" between observed vibratory Doppler velocity undulations, and perhaps a further differentiation of "strong" - vibratory effect (where vibration waves sharply extend from baseline to edge of waveform - see Figure 4 B) vs. "faint" - vibratory effect, (where vibration waves present as mere "feathers" visible upon the top edge of the waveform -see Figure $2 \mathrm{C}$ ) is all we could go by. Eventually, a head to head comparison between compression and vibration regarding safety and efficacy (or both in combination), and methods for statistically comparing them will be needed.

In the future, we would also suggest use of a commercially available TCD head-frame which enables simultaneous Doppler recording of the intra-cranial arteries from both sides.

\section{Conclusions}

In 11 healthy middle aged subjects carotid compressions $(5 \mathrm{~Hz}$ to $8 \mathrm{~Hz},<=1 \mathrm{~cm})$ reliably transmitted flow pulses to $21 / 21$ ipsilateral and $5 / 5$ contralateral intra-cranial arteries interrogated. In 10 subjects carotid vibration ( $30 \mathrm{~Hz} \sim 1 \mathrm{~mm}$ ) transmitted flow pulses to $18 /$ 20 ipsilateral and 4 / 5 contralateral intra-cranial arteries interrogated, however biofeedback was commonly required to ensure device positioning. Mean intra-cranial flow velocities did not statistically differ between compressed vs. non-compressed states. Carotid VC appears feasible for animal testing as a potential reperfusive treatment in AIS, but safety aspects including risks of; ICH, secondary embolus, carotid dissection, and hemodynamic decompensation from carotid sinus pressure, should be vigilantly addressed in future work. Assessment of the more proximal CCA may be considered as an alternative administration site in avoidance of the coronary sinus.

\section{Acknowledgments}

We thank Arianna Hoffmann for her assistance in photography

\section{Conflict of Interest}

Andrew Hoffmann owns shares in Ahof Biophysical Systems Inc., which holds patents relating to vibration in acute stroke. Otherwise no conflict of interest declared. Harjit Gill has received research grants from Ahof Biophysical Systems Inc. Otherwise no conflict of interest declared.

\section{Disclaimer}

The Author's cannot warrant the safety or effectiveness of VC therapy to the carotid or any artery of the neck in a patient at risk for, or having symptoms consistent with acute ischemic stroke. Pre-clinical and then (if warranted) clinical trials are needed to establish the risks versus benefits of this proposed technique.

\section{References}

Aaslid R (1986). Transcranial Doppler examination techniques. In: Aaslid R, ed Transcranial Doppler Sonography. New York 39-59.

Guan J, Zhang S, Zhou Q, Li C, Lu Z (2013). Usefulness of Transcranial Doppler Ultrasound in Evaluating Cervical-Cranial Collateral Circulations. Interv Neurol 2: 8-18.

Jawad Naqvi, Kok Hooi Yap, Gulraiz Ahmad, Jonathan Ghosh (2013). Review ArticleTranscranial Doppler Ultrasound: A Review of the Physical Principles and Major Applications in Critical Care. Hindawi Publishing Corporation. Int Journ Vasc Med 13 pages.

Khaffaf N, Karnik R, Winkler WB, Valentin A, Slany J (1994). Embolic stroke by compression maneuver during transcranial Doppler sonography. Stroke 25: 1056-1057.

Beal MF, Park TS, Fisher M (1981). Cerebral atheromatous embolism following carotid sinus pressure. Arch Neurol 38:310-312.

Bastulli JA, Orlowski JP(1985). Stroke as a complication of carotid sinus massage. Crit Care Med 13: 869.

Friedman SG (1990). Transient ischemic attacks resulting from carotid duplex imaging. Surgery 107:153-155.

Lupetin AR, Davis DA, Beckman I, Dash N (1995). Transcranial Doppler sonography. Part 1. Principles, technique, and normal appearances. Radiographics 15:179-191.

(1995) The National Institute of Neurological Disorders and Stroke rt-PA Stroke Study Group. Tissue plasminogen activator for acute ischemic stroke. N Engl J Med 333:1581-1587.

Hacke W, Kaste M, Bluhmki E, Brozman M, Dávalos A, et al.( 2008) for the ECASS Investigators. Thrombolysis with alteplase 3 to 4.5 hours after acute ischemic stroke. N Engl J Med 359: 1317-1329.

Pagola J, Ribo M, Alvarez-Sabin J, Lange M, Rubiera M, et al. (2007). Timing of Recanalization After Microbubble-Enhanced Intravenous Thrombolysis in Basilar Artery Occlusion. Stroke 38: 2931-2934.

Saqqur M, Tsigvoulis G, Nicoli F, Skoloudik D, Sharma VK, et al. (2014). The Role of Sonolysis and Sonothrombolysis in Acute Ischemic 
Stroke: A Systematic Review and Meta analysis of Randomized Controlled Trials and Case Control Studies. Journal of Neuroimaging 24: 209-220.

Alexandrov, Molina CA, Grotta JC, Garami Z, Ford SR, et al. (2004). for the CLOTBUST Investigators. Ultrasound Enhanced Systemic Thrombolysis for Acute Ischemic Stroke. N Engl J Med 351: 2170-2178.

Eggers J, Koch B, Meyer K, Konig I, Seidel G. (2003) Effect of ultrasound on thrombolysis of middle cerebral artery occlusion. Annals of Neurology. 53: 797-800.

Daffertshofer M, Gass A, Ringleb P, Sitzer M, Sliwka U, et al. (2005). Transcranial Low-Frequency Ultrasound-Mediated Thrombolysis in Brain Ischemia. Increased Risk of Hemorrhage With Combined Ultrasound and Tissue Plasminogen Activator: Results of a Phase II Clinical Trial. Stroke 36: 1441-1446.

Pfaffenberger S, Devcic-Kuhar B, Kollmann C, Kastl SP, Kaun C, Speidl WS,et al.( 2005). Can a commercial diagnostic ultrasound device accelerate thrombolysis? An in vitro skull model. Stroke 36: 124-128.

Hoffmann A, Gill H. (2012) Externally Applied Vibration at 50 Hz Facilitates Dissolution of Blood Clots In-Vitro Am. J. Biomed. Sci 4: 274-284.

Yohannes FG, Hoffmann AK (2008). Non-invasive low frequency vibration as a potential adjunctive treatment for heart attack and stroke. An in-vitro flow model. J Thromb Thrombolysis 25:251-258.

Wobser E, Stumpff U (1978). Intragastral disintegration of blood coagula by mechanical vibration. Endoscopy 10:15-19.

Folts J (1991). An In Vivo Model of Experimental Arterial Stenosis, Intimal Damage, and Periodic Thrombosis. Circulation 83:6-14.

Hoffmann A, Gill H (2012). Diastolic timed Vibro-Percussion at $50 \mathrm{~Hz}$ delivered across a chest wall sized meat barrier enhances clot dissolution and remotely administered Streptokinase effectiveness in an in-vitro model of acute coronary thrombosis. Thrombosis Journal $10: 23$.

Koiwa Y, Honda H, Takagi T, Kikuchi J, Hoshi N,et al. (1997). Modification of human left ventricular relaxation by small-amplitude, phasecontrolled mechanical vibration on the chest wall. Circulation 95:156-162.

Thomas J, Cook DJ, Brooks D (1995). Chest physical therapy management of patients with cystic fibrosis: a meta analysis. Am J Respir Crit Care Med 151:846-850

Koiwa Y, Hashiguchi R, Ohyama T, Isoyama S, Satoh S, Suzuki H et al. (1986). Measurement of instantaneous viscoelastic properties by impedance-frequency curve of the ventricle. Am J Physiol 250:672-684.

Hashiguchi R, Koiwa Y, Ohyama T, Takagi T, Kikuchi J, et al. (1988). Dependence of instantaneous transfer function on regional ischemic myocardial volume. Circ Res 63:1003-1011.

Smith D, Ishimitsu T, Craige E (1984). Mechanical vibration transmission characteristics of the left ventricle implication with regard to auscultation and phonocardiograph. J Am Coll Cordiol 4:3:517-521.

Farber JJ, Purvis JH (1963). Conduction of cardiovascular sound along arteries. Circ Res 12:308-316.

Hancil V, Rod V, Rehakova M (1978). Mass transfer cell with vibrational mixing. Chem Eng J 16:51-56.

Neild A, Ng T, Sheard G, Powers M, Oberti S (2010). Swirl mixing at microfluidic junctions due to low frequency side channel fluidic perturbations. Sensors and Actuators B: Chemical 150:811-818.

Oberti S, Neild A, Ng T (2009). Microfluidic mixing under low frequency vibration. Lab Chip 435-1438.

Marzencki M, Kajbafzadeh B, Khosrow-khavar F, Kaminska B, Menon C (2012). Accelerating Reperfusion with Low Frequency Vessel Deformation during Myocardial Infarction. Computing in Cardiology 9:737-740.

Hoffmann A Inventor (2010). Ahof Biophysical Systems Inc., assignee. Vibration method for clearing acute arterial thrombotic occlusions in the emergency treatment of heart attack and stroke.

Ahof Biophysical Systems Inc: Ethics Review Board and Safety Committee (2014). 3858 Regent St. Burnaby, B.C., Canada V5C 4G8

Arko F, Davis C, Murphy E, Smith S, Timaran C,et al. (2007). Aggressive Percutaneous Mechanical Thrombectomy of Deep Venous Thrombosis. Arch Surg 142:513-519.

Margulis M, Grundel L (1982). Physiochemical processes induced by low-frequency acoustic vibrations in liquids. I. Growth and Pulsation of Gas Bubbles. Russian Journal of Physical Chemistry 56: 876-878.

Griesinger HR, Hiller WJ, Kowalewski TA, Kreter F (1989). Vibration-induced current fields and cavitation effects on model systems for root canal preparation. Zahnarztliche Praxis 40: 213-217.

Krejza J, Arkuszewski M, Kasner S, Weigele J, Ustymowicz A, et al. (2006). Carotid Artery Diameter in Men and Women and the Relation to Body and Neck Size. Stroke 37: 1103-1105.

Lacerda G, Pedrosa R, Lacerda RC, Santos M, Brasil A, Siqueira-Filho A (2009). Complications related to carotid sinus massage in 502 ambulatory patients. Arq Bras Cardiol 92:78-87.

Jatuzis D, Zachrisson H, Blomstrand C, Ekholm S, Holm J, et al. (2000). Evaluation of posterior cerebral artery blood flow with transcranial Doppler sonography: value and risk of common carotid artery compression. Clin Ultrasound 28:452-460.

Kai Y, Morioka M, Yano S, Nakamura H, Makino K,et al. (2007). External Manual Carotid Compression is Effective in Patients with Cavernous Sinus Dural Arteriovenous Fistulae. Interv Neuroradiol 13:115-122. 
Higashida RT, Hieshima GB, et al. (1986). Closure of carotid cavernous sinus fistulae by external compression of the carotid artery and jugular vein. Acta Radiol Suppl 369:580-583.

Vellimana AK, Kadkhodayan Y, Rich KM, Cross DT 3rd, Moran CJ, et al. (2013). Symptomatic patients with intraluminal carotid artery thrombus: outcome with a strategy of initial anticoagulation. J Neurosurg 118:34-41.

Arboixa A, Alióc J (2010). Cardioembolic Stroke: Clinical Features, Specific Cardiac Disorders and Prognosis. Curr Cardiol 6:150-161.

Arenillas JF (2011). Special Reports Intracranial Atherosclerosis Current Concepts. Stroke 42:S20-S23.

Bum JK, Jong SK (2014). Ischemic Stroke Subtype Classification: An Asian View point. Journal of Stroke 16:8-17.

Deepak SM, Jenkins NP, Davidson NC, Bennett DH, Mushahwar SS (2005).Ventricular fibrillation induced by carotid sinus massage without preceding bradycardia. EP Europace 7:638 - 640.

Gurwitz A, Gore J, Goldberg R, Barron H, Breen T, et al. ( 1998). Risk for intracranial hemorrhage after tissue plasminogen activator treatment for acute myocardial infarction. Participants in the National Registry of Myocardial Infarction 2. SOAnn Intern Med 129:597.

Han J, Wong K (2008). Is Counterpulsation a Potential Therapy for Ischemic Stroke? Cerebrovasc Dis 26:97-105.

Han J, Leung T, Lam W, Soo Y, Alexandrov A W, et al. (2008). Preliminary Findings of External Counterpulsation for Ischemic Stroke Patient With Large Artery Occlusive Disease. Stroke 39:000-000.

Alexandrov A W, Liebeskind D, Raman R, Alexandrov A V, Hemmen T, Shahripour R, et al. (2004). Abstract T P51: Tolerability and Safety of External Counterpulsation (ECP) in Acute Ischemic Stroke: Final Results of the Counterpulsation to Upgrade Forward Flow in Stroke (CUFFS) Trial. Stroke ATP51.

Lin W, Han J, Chen X, Xiong L, Leung HW, et al. (2013). Predictors of good functional outcome in counterpulsation-treated recent ischaemic stroke patients. BMJ Open 20: 3.

Li X, Han J, Lin W, Chen X, Leung Tet al. (2014). Abstract W P55: The Degree of Cerebral Blood Flow Augmentation by External Counterpulsation Correlates With Clinical Outcome After Acute Ischemic Stroke. Stroke 45:AWP55.

Haneline MT, Croft AC, Frishberg BM (2003). Association of internal carotid artery dissection and chiropractic manipulation. Neurologist 9:35-44.

Cassidy JD, Boyle E, Cote P, He Y, Hogg-Johnson S, et al. (2008). Risk of vertebrobasilar stroke and chiropractic care: results of a population-based case-control and case-crossover study. Spine 15:176-183.

Wynd S, Westaway M, Vohra S, Kawchuk G (2013). The Quality of Reports on Cervical Arterial Dissection following Cerivical Spinal Manipulation 8 : e59170.

Biffl WL, Moore EE, Ryu RK, Offner PJ, Novak Z, Coldwell DM, et al. (1998). The Unrecognized Epidemic of Blunt Carotid Arterial Injuires. Early Diagnosis Improves Neurologic Outcome. Annals of Surgery $228: 462-470$.

Norris JW, Beletsky V, Nadareishvili ZG (2000). on behalf of the The Canadian Stroke Consortium. Sudden neck movement and cervical artery dissection. CMAJ 163: 38-40.

DeSouza RM, Crocker MJ, Haliasos N, Rennie A, Saxena A (2011). Blunt traumatic vertebral artery injury: a clinical review. Eur Spine 20:1405-1416.

Baron J, Mosely M (2000). For how long is brain tissue salvageable? Imaging-based evidence. J of Stroke \& Cereb. Vasc Dis 9:15-20.

Silke W, Kostopoulos P, Haass A, Keller I, Lesmeister M, et al. (2012). Diagnosis and treatment of patients with stroke in a mobile stroke unit versus in hospital: a randomized controlled trial. The Lancet Neurology 11:397-404.

Belz GG (1995). Elastic properties and Windkessel function of the human aorta. Cardiovasc Drugs Ther $9: 73-83$.

Maloney-Hinds C, Petrofsky JS, Zimmerman G, Hessinger DA (2009). The role of nitric oxide in skin blood flow increases due to vibration in healthy adults and adults with type 2 diabetes. Diabetes Technol Ther 11: 39-43.

Pei Z, Chen J, Zhu M, Liu J, Zhang Q (2004). The effects of infrasound on the secretion of the nitric oxide in rat plasma and the expression of VEGF in vascular endothelia. Chinese Heart Journal 1: 20-22.

Aelen P, Uryash A, Woerlee P, Adams J (2010). Abstract 201: in-vivo response of eNOS up-regulation by acoustic induced vibrations in rats. Circulation 122: A201.

Jahan R, Stewart D, Vinters H, Yong W, Vinuela F, et al. (2008). Middle cerebral artery occlusion in the rabbit using selective angiography: application for assessment of thrombolysis. Stroke 39: 1613-1615.

Menon C, Hoffmann A (2008). Inventors. Simon Fraser University, Assignee. Randomic Vibration for Treatment of Blood Flow Disorders. United States.

Antic S, Galinovic I, Lovrencic-Huzjan A, Vukovic V, Juracic M, et al. (2008). Music as an Auditory Stimulus in Stroke Patients Coll. Antropol 32: 19-23.

Lindblad LE, Lorenz RR, Shepherd JT, Vanhoutte PM (1986). Effect of vibration on canine cutaneous artery. Heart Circ Physiol 19:519-523.

Ljung B, Silvertsson R (1975). Vibration-induced inhibition of vascular smooth muscle contraction. Blood Vessels 12:38-52.

Hudlicka O, Wright A (1978). The effect of vibration on blood flow in skeletal muscle in rabbit. Clin Sci Mol Med 55:471-476.

Takashima H, Tsuruta H, Higashi T, Watanabe M, Isomoto A, et al.( 1987). Effects of mechanical force on blood fibrinolytic activity. Thromb Haemost 58: A550. 